\title{
Evaluación de función pulmonar: ¿qué estudios y a quién?
}

\author{
Pulmonary function evaluation: What studies and to whom? \\ Alejandro Rojas-Montaño* \\ * Nefrólogo de Trasplantes. Hospital Ángeles Pedregal, CDMX.
}

\section{ESTUDIO DE LA PAREJA \\ DONANTE-RECEPTOR PREVIO \\ AL TRASPLANTE RENAL}

Evaluación de la función pulmonar

en el candidato a trasplante renal

En todos los pacientes candidatos a trasplante renal (TR) consideramos como obligatorio se realice una evaluación de la función pulmonar, cuyo objetivo primordial será la búsqueda de factores de riesgo para complicaciones pulmonares postquirúrgicas.

Es obligatorio realizar una búsqueda de factores de riesgo modificables para complicaciones pulmonares postquirúrgicas y establecer intervenciones de manejo; entre los más importantes se encuentran:

- Tabaquismo

- Obesidad

Es obligatorio realizar una búsqueda intencionada de patologías pulmonares cuyo curso pueda complicarse con el procedimiento quirúrgico y/o que afecten la supervivencia del paciente a corto y mediano plazo, independientemente de la enfermedad renal y la función del injerto renal. Entre las más importantes se encuentran:

- Hipertensión arterial pulmonar (HAP).

- Enfermedad pulmonar obstructiva crónica (EPOC).

- Asma.
- Fibrosis pulmonar.

- Enfermedades pulmonares restrictivas.

Sugerimos que los pacientes con las siguientes condiciones respiratorias no sean considerados para trasplante renal:

- Requerimiento crónico de oxígeno intradomiciliario.

- Cor pulmonale severo.

- Asma descontrolada.

- HAP severa no corregible con tratamiento.

- EPOC severo, enfermedad pulmonar restrictiva severa o fibrosis pulmonar con cualquiera de los siguientes parámetros:

a) Volumen espiratorio forzado en un segundo (FEV1) $<25 \%$ del valor predicho.

b) Presión arterial de oxígeno al aire ambiente $<60 \mathrm{mmHg}$ o desaturación con el ejercicio < $90 \%$.

c) Más de cuatro infecciones de vías respiratorias bajas en los últimos 12 meses.

d) Enfermedad moderada con progresión documentada en los últimos meses.

Sugerimos que los pacientes con EPOC moderado, enfermedad pulmonar restrictiva moderada o fibrosis pulmonar con cualquiera de los siguientes parámetros se les considere con contraindicación relativa: 
e) Volumen espiratorio forzado en un segundo (FEV1) entre 25 a $50 \%$ del valor predicho.

f) Presión arterial de oxígeno al aire ambiente < $60-70 \mathrm{mmHg}$ con desaturación con el ejercicio < $90 \%$.

Para la valoración inicial de candidatos a trasplante renal sin antecedentes de enfermedades pulmonares, sin disnea, sin síntomas de falla cardiaca derecha ni desaturación de oxígeno al aire ambiente, el examen paraclínico obligatorio a realizar es la radiografía de tórax en proyección AP y lateral, se sugiere que más estudios como tomografía de tórax, espirometría o medición de gases arteriales sean realizados de acuerdo con la presencia de patologías pulmonares específicas con la intervención del especialista en neumología.

Es deseable que los exámenes para la valoración pulmonar sean realizados cuando el paciente se encuentre sin sobrecarga de volumen.

\section{RAZONES}

Existen pocos datos sobre la valoración pulmonar preoperatoria ideal en los candidatos a TR; sin embargo, se acepta que deban cumplir con los estándares mínimos de la cirugía abdominal en la población general. Deberán buscarse intencionadamente factores de riesgo para complicaciones pulmonares perioperatorias, y uno de los más importantes es el consumo de tabaco. Se ha documentado que los fumadores experimentan significativamente más complicaciones pulmonares postoperatorias que los no fumadores. ${ }^{1}$ En un estudio se encontró que la incidencia de complicaciones pulmonares posteriores a cirugía abdominal en fumadores fue del doble que en los no fumadores y que el consumo de más de 20 cigarros al día se asoció a un incremento de cuatro veces en la aparición de atelectasias postoperatorias. ${ }^{2}$

Por otro lado, el consumo de tabaco se ha asociado a un incremento del riesgo de pérdida del injerto y muerte del paciente. En un estudio se encontró que sujetos con antecedente de consumir al menos 25 paquetes al año, tuvieron un riesgo de más de $30 \%$ de falla del injerto que aquéllos que fumaban menos o no habían fumado; también se encontró que los que habían suspendido el consumo del cigarrillo al menos cinco años antes del trasplante tuvieron un menor riesgo de falla del injerto. Por lo anterior, es aconsejable ofrecer intervenciones para suspender el consumo de tabaco, entre las que pueden encontrarse medidas farmacológicas y no farmacológicas. ${ }^{3}$
Asimismo, un factor de riesgo bien reconocido para complicaciones posteriores al trasplante renal es la obesidad. Desde el punto de vista pulmonar la obesidad conduce a restricción ventilatoria y disminución de los volúmenes pulmonares, mismos que suelen empeorar posterior a la cirugía. La aparición de atelectasias postoperatorias e hipoxemia son más frecuentes en los pacientes obesos que reciben un injerto renal. ${ }^{4,5}$ Las recomendaciones sobre el manejo de la obesidad en el candidato a trasplante renal se discuten con más detalle en el apartado correspondiente de este documento.

Existen patologías pulmonares cuyo curso puede complicarse con el procedimiento quirúrgico y/o que afectan la supervivencia del paciente a corto y mediano plazo, lo cual se convierte en un factor determinante para considerar a un paciente como candidato. Una de las enfermedades más importantes es la HAP, dicha entidad es frecuente en los sujetos con ERC en estadio avanzado. La aparición de HAP se ha asociado con el tiempo en diálisis, disfunción cardiaca sistólica y diastólica, el consumo de tabaco y sobrecarga de volumen. La HAP severa se ha asociado con una disminución en la supervivencia del paciente y del injerto postrasplante. ${ }^{6}$ El estudio de elección para la detección y evaluación inicial de la HAP es el ecocardiograma transtorácico, cabe mencionar que dicho estudio debería realizarse, idealmente, cuando el paciente no presente sobrecarga de volumen, pues la misma es una causa reversible de HAP. En los pacientes con HAP diagnosticada por ecocardiograma es obligatorio que se realice una evaluación más profunda para confirmar el diagnóstico, así como la causa subyacente, la realización de un cateterismo cardiaco derecho deberá considerarse en conjunto con especialistas en cardiología y neumología. ${ }^{7}$ Una vez establecido el diagnóstico, la causa y la severidad de la HAP, deberá instaurarse el manejo apropiado antes de considerarse como candidatos a trasplante renal.

Los pacientes con enfermedades pulmonares crónicas como EPOC, asma, fibrosis pulmonar y enfermedades restrictivas, aun en estado controlado, tienen mayor riesgo de complicaciones pulmonares postoperatorias. La instrumentación de la vía aérea en esos enfermos puede conllevar a la exacerbación del proceso inflamatorio bronquial, con el empeoramiento de la hiperreactividad y un mayor riesgo de broncoespasmo. La colonización bacteriana crónica de las vías aéreas, con el aumento del trabajo respiratorio, también podría contribuir al aumento de complicaciones. ${ }^{8}$

De manera general, el riesgo y la gravedad de las complicaciones postoperatorias son proporcionales al 
grado de compromiso clínico y espirométrico prequirúrgico, clasificándose como moderado si el VEF1 está entre 50 y $80 \%$, y grave si el VEF $1<50 \% .{ }^{9}$ Por lo anterior, los pacientes con dichas condiciones deberán ser valorados por el especialista en neumología para optimizar el manejo de su enfermedad. La severidad de estas enfermedades puede disminuir la supervivencia de los pacientes de forma importante y limitar los beneficios del trasplante renal. En un estudio se documentó que los pacientes que requirieron oxígeno intradomiciliario de modo permanente por diversas patologías pulmonares, mostraron tasas de supervivencia menores al $30 \%$ a seis años, ${ }^{10}$ los pacientes con un FEV $1<40 \%$ del valor predicho mostraron una tasa de supervivencia a seis años de $50 \% .{ }^{11}$ Por lo antes mencionado, sugerimos que los sujetos con condiciones pulmonares graves, que cumplan con los criterios de severidad mencionados anteriormente sean considerados con contraindicación absoluta o relativa para el trasplante renal.

La radiografía de tórax es el estudio mínimo para la valoración inicial de candidatos a trasplante renal sin antecedente de enfermedades pulmonares, sin disnea, sin síntomas de falla cardiaca derecha ni desaturación de oxígeno al aire ambiente. En aquéllos con síntomas de enfermedades pulmonares o padecimientos ya diagnosticados, es obligatorio realizar todos los estudios paraclínicos pertinentes para su valoración y manejo con la intervención del especialista en neumología, entre ellos podrán incluirse la medición de gases arteriales, tomografía de tórax, espirometría, ecocardiograma, cateterismo cardiaco derecho, entre otros. Debido a la influencia de la sobrecarga de volumen en los resultados de los estudios utilizados para la valoración pulmonar es aconsejable sean realizados cuando el paciente se encuentre en peso seco.

\section{Evaluación de la función pulmonar en el potencial donante}

Es obligatorio que todos los pacientes candidatos a ser donantes renales sean sometidos a una evaluación de la función pulmonar, cuyo objetivo será la búsqueda de factores de riesgo para complicaciones o condiciones pulmonares que otorguen un riesgo quirúrgico inaceptable.

Es obligatorio que a todos los candidatos que fumen se les recomiende suspender el consumo de tabaco de manera definitiva y es aconsejable cumplir con al menos cuatro semanas de suspensión de éste antes de la donación para reducir el riesgo de complicaciones pulmonares perioperatorias. Sugerimos que todos sean referidos a un especialista en el manejo de adicciones.

En los sujetos sin antecedentes ni síntomas de enfermedades pulmonares y con saturación de oxígeno al aire ambiente $>90 \%$ mediante oximetría de pulso, la radiografía de tórax es el único estudio de gabinete obligatorio para la valoración pulmonar inicial.

En los pacientes con antecedentes o síntomas de enfermedades pulmonares o con saturación de oxígeno al aire ambiente $<90 \%$, es obligatoria una evaluación más profunda que incluya otros estudios como espirometría, tomografía de tórax o medición de gases arteriales.

Sugerimos que los sujetos con enfermedades pulmonares crónicas con las siguientes características no sean considerados para ser donantes:

- Uso de oxígeno intradomiciliario.

- HAP de cualquier grado.

- Asma moderada o severa, según la clasificación de las guías de la iniciativa global para el asma 2019.

- EPOC moderado, severo o muy severo según la clasificación de GOLD 2019.

Los pacientes con condiciones respiratorias crónicas leves, de pronóstico favorable y sin descontrol documentado en los últimos seis meses podrían ser considerados para ser donantes; sin embargo, cada caso deberá ser estudiado de forma individualizada junto con el especialista en neumología.

\section{RAZONES}

Las complicaciones respiratorias postoperatorias en el donante renal han sido reportadas en $2.5 \%$ de los casos $^{12}$ y las enfermedades pulmonares preexistentes a la donación se han relacionado con un mayor riesgo de reingreso hospitalario a 90 días. ${ }^{13}$ De tal forma que todos los pacientes candidatos a ser donantes renales deberán ser sometidos a una evaluación de la función pulmonar, cuyo objetivo será la búsqueda de factores de riesgo para complicaciones pulmonares o condiciones pulmonares que otorguen un riesgo inaceptable asociado a la donación.

El consumo de tabaco es un factor de riesgo bien documentado para complicaciones postoperatorias, y, por otro lado, confiere múltiples riesgos a largo plazo. Por lo anterior, a todos los potenciales donantes se les deberá recomendar suspender el consumo de tabaco 
de manera definitiva. En un estudio se documentó que el riesgo de presentar complicaciones postquirúrgicas en cirugías abdominales fue menor en quien suspendió el consumo de tabaco con un mínimo de cuatro semanas antes del procedimiento. ${ }^{14}$

La mayoría de las guías internacionales ${ }^{15,16}$ sobre la valoración perioperatoria recomiendan que en los pacientes sin antecedentes ni síntomas de enfermedades pulmonares y con saturación de oxígeno al aire ambiente $>90 \%$, la radiografía de tórax sea el único estudio de gabinete recomendado para la valoración pulmonar inicial. La espirometría, la tomografía de tórax o la medición de gases arteriales deberán ser realizados cuando haya indicación de acuerdo con síntomas.

Por otra parte, las enfermedades pulmonares crónicas como EPOC, asma, hipertensión pulmonar, entre otras, han demostrado elevar el riesgo de complicaciones postquirúrgicas, ${ }^{12}$ según la severidad de las mismas pueden conferir un riesgo inaceptablemente alto para un donador renal, de tal forma que condiciones como el uso de oxígeno intradomiciliario, HAP de cualquier grado, enfermedad pulmonar leve pero con progresión documentada en los últimos meses, asma moderada o severa y EPOC moderado, severo o muy severo, deberían considerarse como contraindicaciones para la donación. Los pacientes con condiciones respiratorias crónicas leves, con un buen pronóstico y sin descontrol documentado en los últimos seis meses podrían ser considerados para ser donantes; sin embargo, cada caso deberá ser estudiado de forma individualizada junto con el especialista en neumología.

\section{REFERENCIAS}

1. Bluman LG, Mosca L, Newman N, Simon DG. Preoperative smoking habits and postoperative pulmonary complications. Chest. 1998; 113: 883-889.

2. Wellman JJ, Smith BA. Respiratory complications of surgery. In: Lubin MF, Walker HK, Smith RB, editors. Medical management of the surgical patient. Boston: Butterworth; 1988. pp. 155-60.
3. Kasiske BL, Klinger D. Cigarette smoking in renal transplant recipients. J Am Soc Nephrol. 2000; 11: 753.

4. Johnson DW, Isbel NM, Brown AM, Kay TD, Franzen K, Hawley $\mathrm{CM}$ et al. The effect of obesity on renal transplant outcomes. Transplantation. 2002; 74: 675-681.

5. Schwarznau A, Matevossian E, Novotny A, Stangl M. Outcome of living donor renal transplantation in obese recipients. Transplant Proc. 2008; 40: 921-922.

6. Issa N, Krowka MJ, Griffin MD et al. Pulmonary hypertension is associated with reduced patient survival after kidney transplantation. Transplantation. 2008; 86 (10): 1384-1388.

7. Frost A, Badesch D, Gibbs JSR, Gopalan D, Khanna D, Manes A et al. Diagnosis of pulmonary hypertension. Eur Respir J. 2019; 53 (1). pii: 1801904.

8. Jaber S, Delay JM, Chanques G, Sebbane M, Jacquet E, Souche B et al. Outcomes of patients with acute respiratory failure after abdominal surgery treated with noninvasive positive pressure ventilation. Chest. 2005; 128: 2688-2695.

9. Ramakrishna G, Sprung J, Ravi BS, Chandrasekaran K, McGoon MD. Impact of pulmonary hypertension on the outcomes of noncardiac surgery: predictors of perioperative morbidity and mortality. J Am Coll Cardiol. 2005; 45: 1691-1699.

10. Hansen EF, Phanareth K, Laursen LC et al. Reversible and irreversible airflow obstruction as predictor of overall mortality in asthma and chronic obstructive pulmonary disease. Am J Respir Crit Care Med. 1999; 159 (4 pt 1): 1267-1271.

11. Chailleux E, Fauroux $B$, Binet $F$ et al. Predictors of survival in patients receiving domiciliary oxygen therapy or mechanical ventilation. A 10-year analysis of ANTADIR observatory. Chest. 1996; 109 (3): 741-794.

12. Mjoen G, Oyen $\mathrm{O}$, Holdaas $\mathrm{H}$ et al. Morbidity and mortality in 1022 consecutive living donor nephrectomies: benefits of a living donor registry. Transplantation. 2009; 88: 1273-1279.

13. Axelrod D, Lentine KL, Schnitzler MA, Luo X, Xiao H, Orandi BJ et al. The incremental cost of incompatible living donor kidney transplantation: a national cohort analysis. Am J Transplant. 2017; 17 (12): 3123-3130.

14. Abecassis $\mathrm{M}$, Adams $\mathrm{M}$, Adams $\mathrm{P}$ et al. Consensus statement on the live organ donor. JAMA. 2000; 284: 2919-2926.

15. Qaseem A, Snow V, Fitterman N et al. Risk assessment for and strategies to reduce perioperative pulmonary complications for patients undergoing non cardiothoracic surgery: a guideline from the American College of Physicians. Ann Intern Med. 2006; 144: 575-580.

16. Clavien PA, Barkun J, de Oliveira ML et al. The Clavien-Dindo classification of surgical complications: five-year experience. Ann Surg. 2009; 250: 187-196.

\section{Correspondencia:}

Dr. Alejandro Rojas-Montaño

E-mail: alex_seg@hotmail.com 